\title{
Efficient System of Laser Diode CW Pumped on Nd:YAG Laser
}

\author{
F. H. Mustafa* \\ M. I. Azzawe** \\ Physics Department, College of Education, Mosul University. **
}

Received

17 / 06 / 2007
Accepted

17 / 07 / 2007

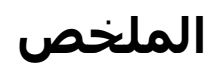

يقدم هذا البحث أكفأ إنجاز في تصميم و بناء منظومة ليزرية مصغرة من الحالة

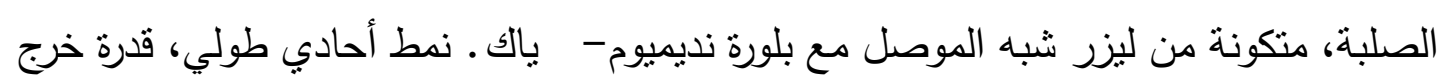

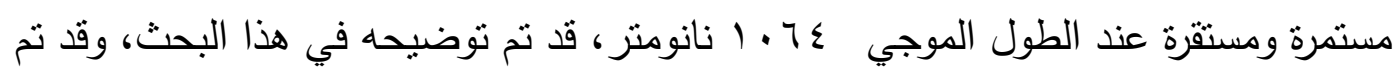

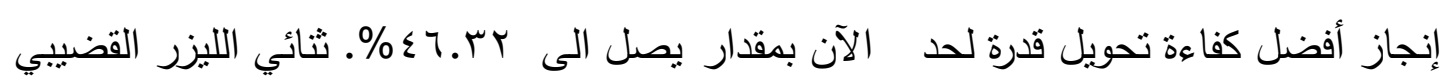

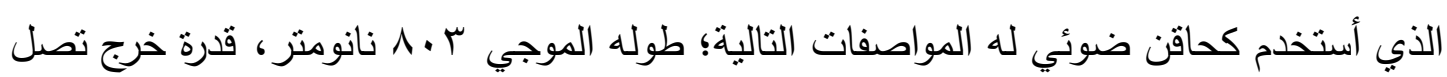

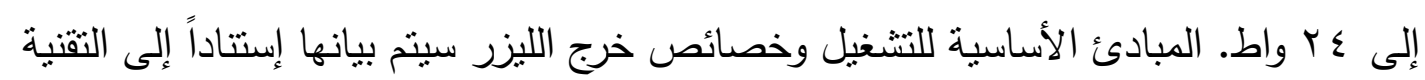

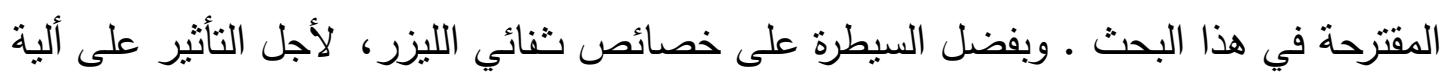

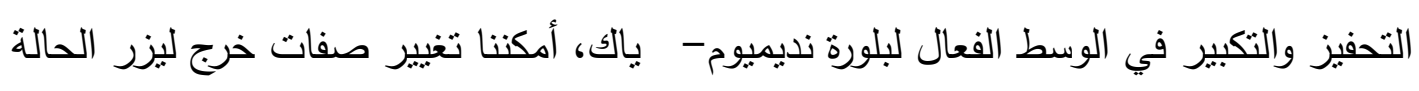
الصلبة وفق صيغ مطلوبة في هذه التجربة.
\end{abstract}

\begin{abstract}
In this paper, we report the recent progress made in the design and development of a miniature solid-state system, consisting from a bar laser diode and a slab of Nd:YAG crystal. Single longitudinal mode, stable $\mathrm{CW}$ power output at $1064 \mathrm{~nm}$ wavelength has been demonstrated. An ever power conversion efficiency was reached with an amount of $46.32 \%$. Bar laser diode of wavelength $803 \mathrm{~nm}$, and power output up to $24 \mathrm{~W}$ was used as a direct pumping device. Basic principles of operation and output characteristics will be demonstrated by this proposed technique. Taking the full advantages of the bar laser diode array, influencing the mechanisms of excitation, amplification, and saturation in the crystal; spectral emission, efficiency and beam quality can be tailored in the experiment.
\end{abstract}

*E-mail: scienc-far@yahoo.com 


\section{Introduction}

$\mathrm{Nd}$ :YAG laser is the promising laser source for many applications in our daily life when it is easy to operate, compact, and low power assumptions. The optical pumping from the laser diode emission can fulfill these requirements. Also, this optical pumping have many other benefits over lamp excitation of the Nd:YAG crystal due to their low power consumption and easy to operate. For many purposes, the Nd:YAG laser offer the bright future of application in medicine, industry, and scientific. Investigation of Corneal ablation using the UV laser pulses from the Q-switched Nd:YAG with the fifth harmonic generation was reported [1]. Diode pumped solid-state laser (Nd:YAG) has entered the market especially in industries for precision cutting and welding [2,3]. During the past few years much work has been done in order to obtain an efficient blue laser from the Nd:YAG laser [4]. This has many interests of applications such as display technologies, high-density optical disk systems and medical diagnosis [5].

CW operation of Nd:YAG laser has been reported recently with a differential efficiency equal to $6.4 \%$, with laser diode array as an optical pumping source [6]. These arrays can be used for direct band pumping, minimizing the amount of quantum defect in the laser system. Besides, the wavelength of the laser diode (LD) can be selected within certain bounds by choosing the appropriate combination of semiconductor materials. Therefore, it is possible to engineer these devices according to the needs of various solid-state laser gain media. This allows pumping efficiently the gain medium without wasting a large amount of energy by heating the crystal [6], and can provide excellent spatial mode quality and narrow linewidth $[7,8]$. The enhancement of overall efficiency, low transverse mode laser emission in miniature lasers, and scaling to high powers are among the most important directions in the development of solid-state lasers. The first of these implies an increase of slope efficiency and a reduction of emission threshold; the second relies on increased absorption of the pump while the third imposes the reduction on heat generation [7]. These difficulties have been overcome in our investigation, as will be illustrated in the article.

This article outline the ever reached differential efficiency of CW operating Nd:YAG laser by optical pumping from the bar laser diode (BLD), and covers the spectral emission of the power output maintained in the fundamental mode with high beam-quality. This requirement is very crucial in optical pumping [9,10]. The schematic layout of the Nd:YAG laser with BLD was assembled in a novel manner to provide a high electrical-to-optical conversion efficiency. 


\section{Layout of the Laser System}

Fig.(1) shows the schematic diagram of the proposed laser system carried out in the experiment consisting mainly from; the BLD assembly [11], coupling micro-lens array, the Nd:YAG crystal, and the output mirror. The BLD has the following characteristics; wavelength $803 \mathrm{~nm}$, power output up to $24 \mathrm{~W}$, high external quantum efficiency of 1.66 , and a threshold current of $7.4 \mathrm{~A}$.

Micro-lens array (MLA); $12 \mathrm{~mm}$ length, $1.5 \mathrm{~mm}$ thicknesses and focal length of equal to $4 \mathrm{~mm}$, was used to reduce the divergence of BLD and to collect it to the first face of Nd:YAG crystal. The objective of the MLA is the to collimate the light from the BLD and to focus it on the end of sample (Nd:YAG).

A Neodymium: Yttrium Aluminum Garnet (Nd:YAG) crystal, with dimensions $(12 \times 1.5 \times 4) \mathrm{mm}^{3}$, wavelength $1064 \mathrm{~nm}$ and maximum output power of $3 \mathrm{~W}$ used in the system. YAG crystal was doped with the Neodymium $\left(\mathrm{Nd}^{+3}\right)$ ions of $(1-1.1 \%)$. The crystal faces were coated in two sides $S_{1}$ and $S_{2}$. The side $S_{1}$ has high transmission for pumping radiation $807 \mathrm{~nm}$ and high reflectivity for the generated radiation. The other side $S_{2}$ has an antireflection (AR) coating for the $1064 \mathrm{~nm}$.

The output mirror Bk7 has its faces coated in two sides $S_{1}$ and $S_{2}$. The side $S_{1}$ is a concave mirror with $250 \mathrm{~mm}$ radius and had high reflectivity at $1064 \mathrm{~nm}$. The side $S_{2}$ is a convex mirror with $86 \mathrm{~mm}$ radius with a partial transmission coating (90\% at $1064 \mathrm{~nm})$.

To direct maximum radiation from pumping source (BLD) to the $\mathrm{Nd}$ : YAG crystal, a level adjustment system was used. The Nd:YAG crystal placed over the level adjustment system, which consisted of a thin Aluminum layer, placed on the Copper block, and fixed on the heat sink by means of two screws, that can move the crystal "up and down".

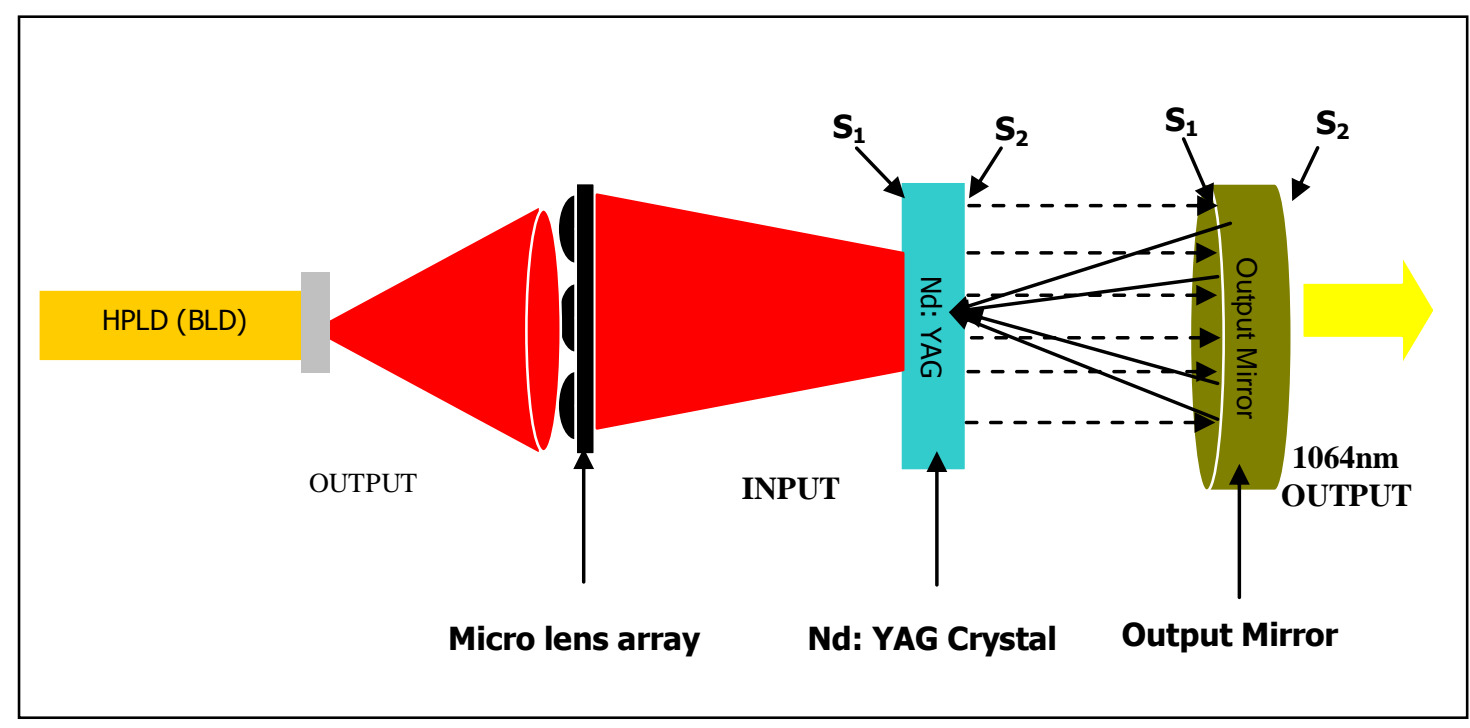

Fig.(1):Layout of the laser system proposed in this investigation. 


\section{Experimental Results}

The main principle measurements that has to be carried out for the laser resonator proposed in the investigation, is the power output of the solid-state laser versus the input power of the BLD, as shown in Fig.(2), the characteristic curve of these measurements. Threshold power was found by a linear regression of the stimulated emission as $714 \mathrm{~mW}$ for the BLD and power conversion efficiency of $46.32 \%$. The differential efficiency (slope efficiency), which is defined as the amount of increase in signal power of the Nd:YAG per unit increase in pump power of the BLD above threshold, has been called here as power conversion efficiency. This efficiency is ever now been reached for laser diode pumping Nd:YAG laser (to our knowledge). The high electrical-to-optical conversion efficiency is due to the novel mount and stability of the power output of the BLD, and the excellent optical coupling achieved in the experiment. The temperature of the assembly (BLD plus the Nd:YAG crystal) have been kept constant during the measurements by an electronic circuitry with the aid of thermoelectric cooler and liquid Nitrogen (c.f. Ref.[11]).

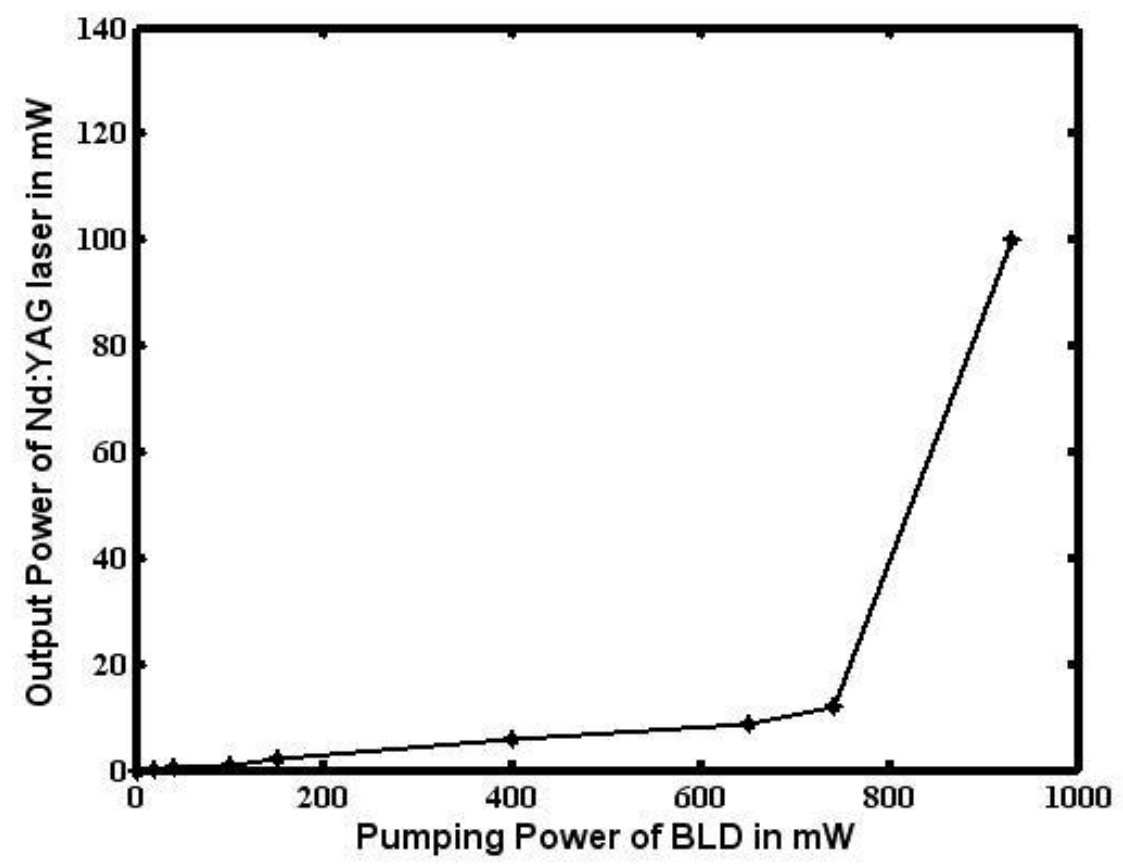

Fig.(2): Nd:YAG laser (1064 $\mathrm{nm})$ power output as a function of BLD pump power $(803 \mathrm{~nm})$. 
The no output power below the threshold pump power and a linear increase in power after crossing this threshold value (similar to the behavior of laser diodes) has been nicely confirmed in Fig.(2). Threshold pump power was determined to be $714 \mathrm{~mW}$.

To investigate the dependence of the power output of the Nd:YAG laser on the temperature of the crystal mount, while keeping the pump power as extremely fixed, we have obtained the following results shown by Fig.(3a). The plot shows a sharp decrease in the power with increasing temperature. In order to assess a relation of this dependence, we have plotted the first derivative of the power output with respect to temperature and plotted the calculated results against the corresponding temperature as in Fig.(3b). These calculations were performed with Matlab package and the following quadratic equation was obtained with curve- fitting tools provided with the package:

$$
\frac{d P}{d T}=-0.0042 \cdot x^{2}+0.39 . x-11
$$

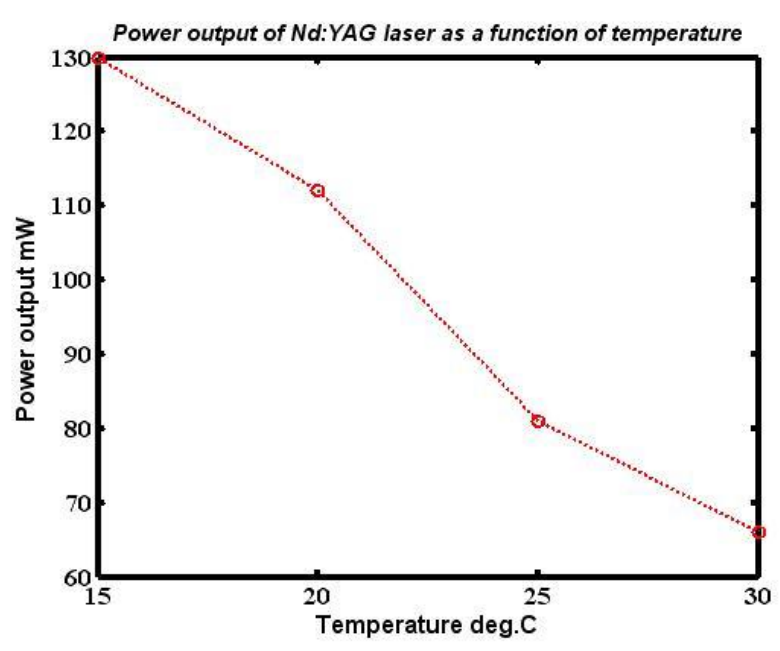

(a)

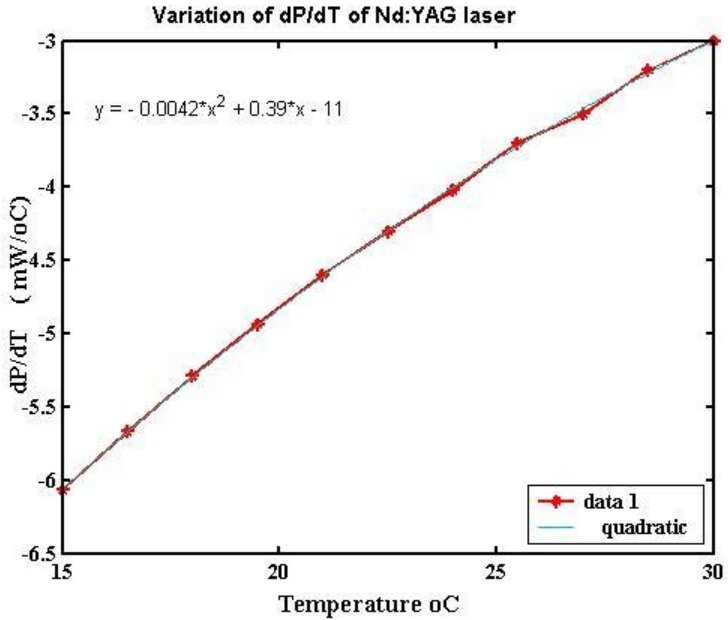

(b)

Fig.(3): Variation of Nd:YAG power output with temperature while keeping the input pumping power of the BLD as extremely constant.

(a): Power versus temperature,

(b): First derivative of power with respect to temperature against temperature. 
The interpretation of Eqn.(1) is that, the power efficiency is decreasing with increasing temperature quadratically and suggesting to be more sensitive to any variation of the mount temperature. This is true since the laser is operating continuously rather than pulsed, as the case for any solid-state laser.

The next crucial property of the laser system is the spectral output, i.e., the wavelength of the $\mathrm{Nd:YAG}$ variation with the input pump power (BLD) and with also the temperature. To assure the optimization of the mode overlapping between the pump and the fundamental cavity mode inside the gain medium while increasing the pump power or the temperature, both the BLD and the Nd:YAG spectra have been monitored instantly at $20{ }^{\circ} \mathrm{C}$, as shown in Fig.(4). This figure represents the mode structure of the BLD output, Fig.(4a),. The emission spectrum generated from the BLD was absorbed by the Nd:YAG crystal without wasting a large amount of energy by heating the crystal. Fig.(4b) shows the typical spectral emission of the $1.064 \mu \mathrm{m}$ laser. The output of Nd:YAG laser was directed to the input of a monochromator (MP-1018B) and was adjusted with an entrance slit and proper speed scan rate of. The output of the monochromator was received by a photodiode that was placed in front of the exit slit. The photodiode output was then connected to a chart recorder via a preamplifier of $(2 \mathrm{~V})$ full scale and $(2 \mathrm{~cm} / \mathrm{min})$ paper advance rate.

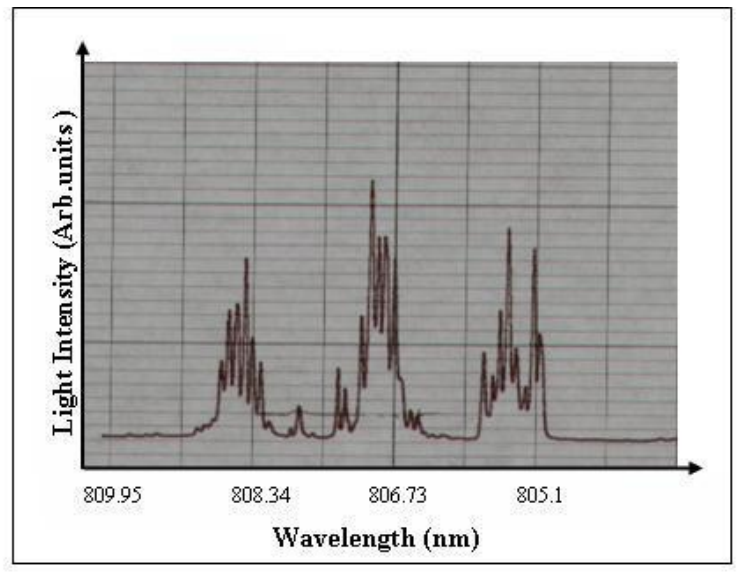

(a)

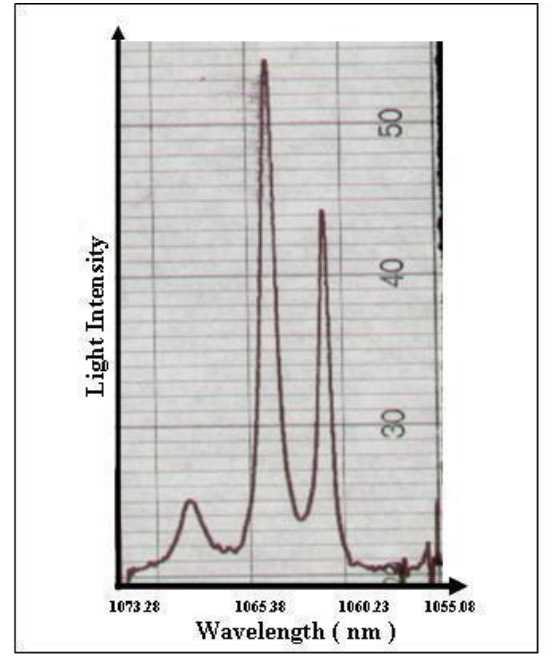

(b)

Fig.(4): Spectral emission of (a) BLD and (b) Nd:YAG laser at $20^{\circ} \mathrm{C}$.

The spectral output of the Nd:YAG laser were recorded as a function of pumping power of the BLD as illustrated in Fig.(5), with temperature as being kept constant at $25^{\circ} \mathrm{C}$ during the measurements. 


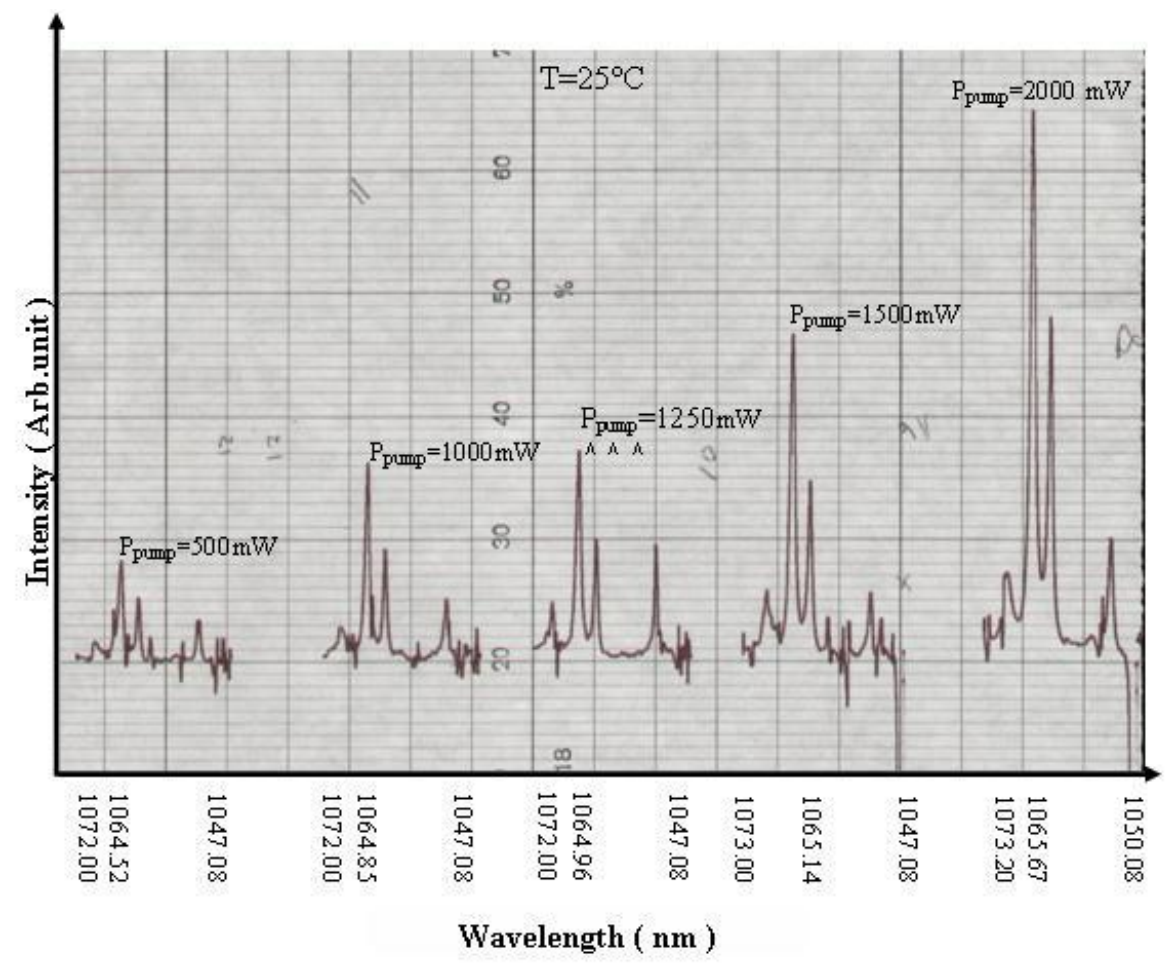

Fig.(5):Spectral dependence of Nd:YAG laser on the pump power of the BLD, at $25^{\circ} \mathrm{C}$.

In order to assess the variation of peak wavelength of the Nd:YAG laser spectra with the pump power, we have calculated the change in wavelength with pump power and plotted the results as in Fig.(6).

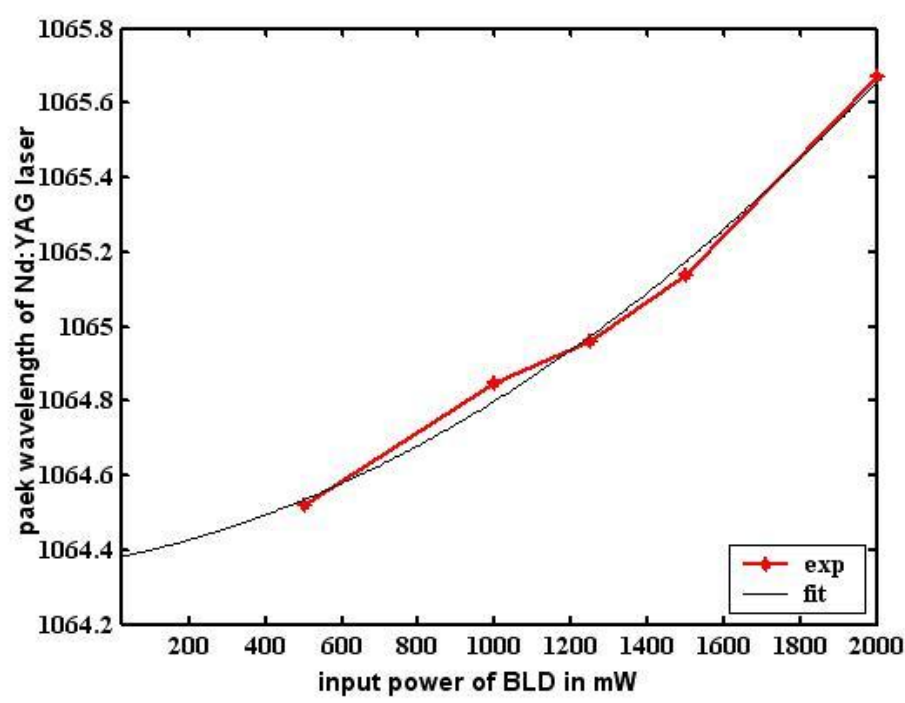

Fig.(6):Dependence of wavelength of Nd:YAG laser on the pump power of BLD, with constant temperature $\left(25^{\circ} \mathrm{C}\right)$. 
It is obvious from the graph that the change is not linear, and the curve-fitting tools of the Matlab give the following equation:

$$
\lambda(n m)=2.17 \times 10^{-7} \text { Pin. }^{2}+2.1 \times 10^{-4} \text { Pin }+1064 .
$$

With Pin is the input power of the BLD laser in $\mathrm{mW}$. This equation provides us with the estimated wavelength at a given input power. If we neglect the first term of the equation, just for instance to make the variation as linear, we get a value of $\left(2.1 \times 10^{-4} \mathrm{~nm} / \mathrm{mW}\right)$ change in wavelength $(\mathrm{nm})$ per $(\mathrm{mW})$ change in input power. This is a neglectable variation in wavelength compared to its wavelength; if threshold power has been reached in very variation.

To study the peak wavelength dependence on the operating temperature, we have obtained the results as shown in Fig.(7). The mount temperature was varied with extreme caution with aid of liquid nitrogen, to allow a stable power output for both of the lasers. The spectral variation of Nd:YAG was monitored instantly with the monochromator while keeping the input pump power constant at $1500 \mathrm{~mW}$. The graph shows that the variation in the output power of $\mathrm{Nd}$ :YAG laser had decreased noticeably. In addition to the decrease of the power output, we can notice that the longitudinal mode had not been deformed due to temperature rise. The peak shift in the laser wavelength has to be related to the thermally induced effects that have been accompanied with the temperature rise, and to the gain-temperature relation in the laser cavity.

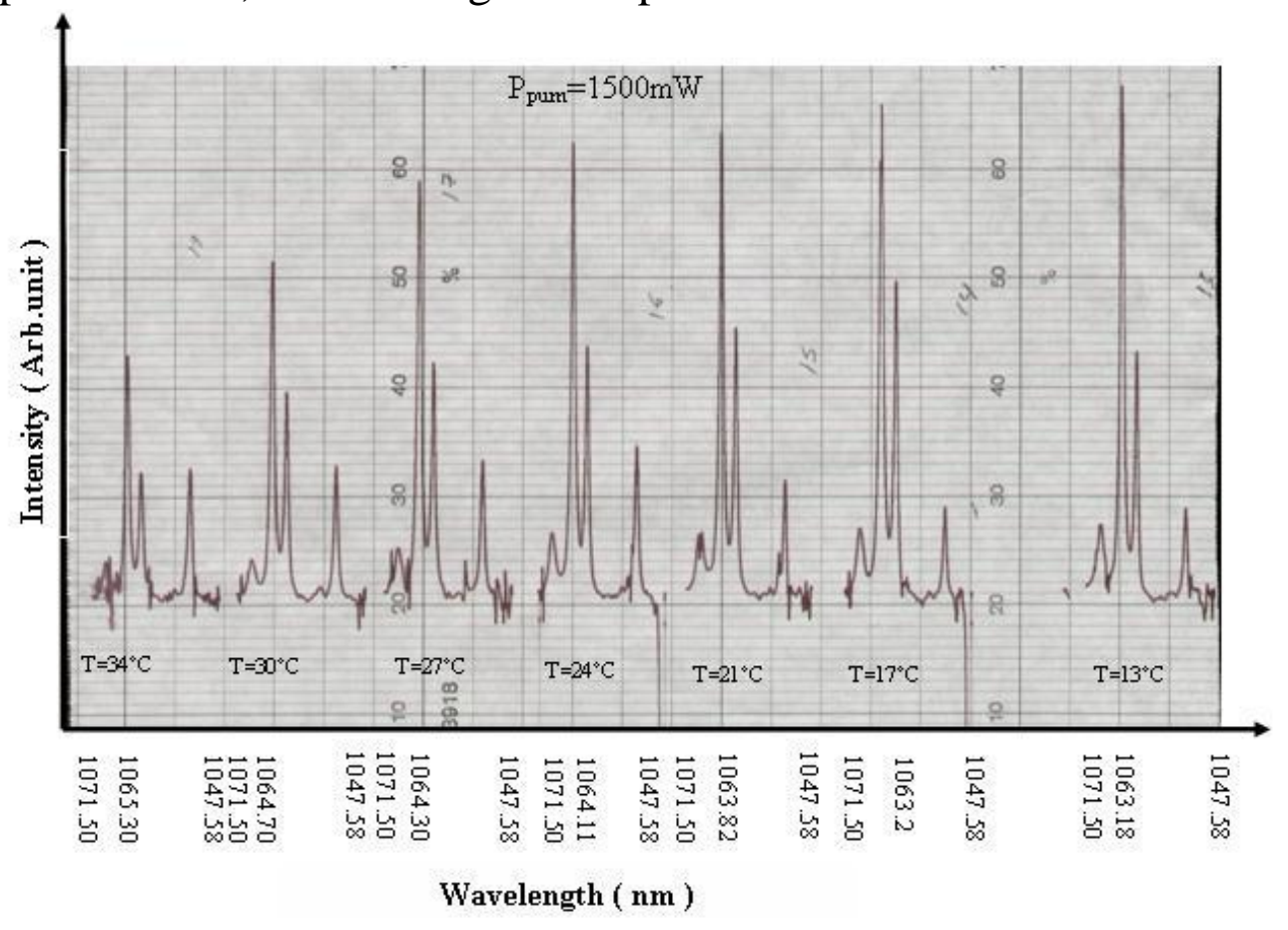

Fig.(7): The effect of temperatures on the spectral emission of the Nd:YAG laser. Pump power was kept constant at $1500 \mathrm{~mW}$, well above threshold during the measurements. 
After evaluating the variation in peak wavelength shift with temperature, the results were then plotted as in Fig.(8). It is worth stating that, the shift is nonlinear corresponding to the nonlinear effect on the gain medium of the laser, suggesting a gain-temperature nonlinear formula inherited by the Nd:YAG laser.

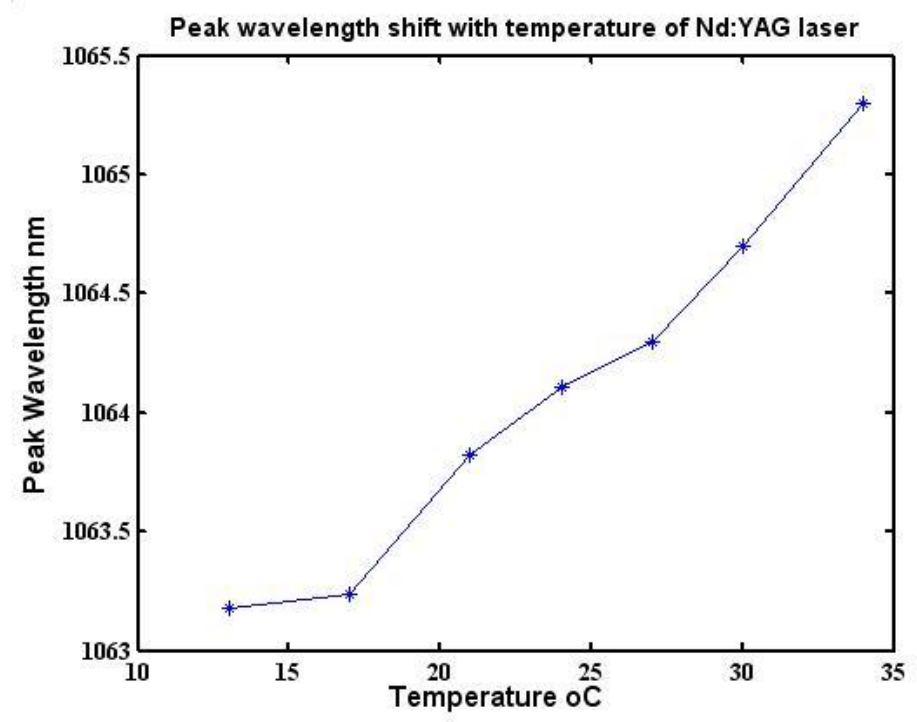

Fig.(8): Peak wavelength shift with temperatures of the Nd:YAG laser, while keeping the pump power constant at $1500 \mathrm{~mW}$ during the measurements.

\section{Conclusions}

Thanks for the advent of the bar laser diode, which made the ability to operate the Nd:YAG laser continuously with good efficient. The operation characteristics of the proposed system had been studied, namely; the power output dependence on the input power, temperature, and the influence of the operating parameters on the spectral emission. The wavelength shift with both the temperature of the mount, or the launching power of the BLD had a neglectable effect. This is due the efficient temperature control that had been built for this purpose. The dependence of the power output of the Nd:YAG laser on the temperature of the crystal mount, had shown a critical effect on the power conversion efficiency and the power output of the solid-state laser. This is due the influence of the extra heating by the crystal that waste the input energy for the gain medium and the thermally induced effects. In order to improve the characteristics of the system, a multiple antireflection-coated array lens has to be used to minimize the quantum effects defects in the laser system. A desired wavelength, pulse operation, with any repetition rate and pulse duration, and beam quality suitable for any requirements can be fulfilled by this system. 


\section{References}

1. Dair, G. T., Pelouch, W. S., van Saarloos, P. P., Lloyd, D. J., Paz Linares, S. M., and Reinholz, F., IOVS Reports Vol. 40, No.11, 2752, (1999).

2. Poprawe, R., and Schulz, W., RIKEN Rev. 50, 3 (2003).

3. Lee, S., Choi, D. W., and Cha, B. H., Korean J. Phys. Soc. 49, 430 (2006).

4. Pavel, N., Shoji, I., and Taira, T., Opt. Laser Tech. 36, 581(2004).

5. Langrock, C. et al., IEEE J. Select. Top. Quant. Electron. XX,101(2002).

6. Shoji, I. et al., Opt. Lett. 27, 234 (2002).

7. Lupei, V., Pavel, N., and Taira, T., Appl. Phys. Lett. 80, 4309 (2002).

8. Lupei, V., Pavel, N., and Taira, T., T., IEEE J. Quant. Electron. 38, 40, (2002).

9. Ostermeyer, M., Kappe, P., Menzel, R., and Wulfmeyer, V., Appl. Opt. 44, 582 (2005).

10. Henriksson, M., Tiihonen, M., Pasiskevicus, V., and Laurell, F., Opt. Lett. 31, 1878 (2006).

11. Mustafa, F. H., and Azzawe, M. I., مبول للنشر في مجلة التربية والعلم 